\title{
Fractionating the Default Mode Network: Distinct Contributions of the Ventral and Dorsal Posterior Cingulate Cortex to Cognitive Control
}

\author{
Robert Leech, ${ }^{1}$ Salwa Kamourieh, ${ }^{1}$ Christian F. Beckmann, ${ }^{1,2}$ and David J. Sharp ${ }^{1}$ \\ ${ }^{1}$ Computational, Cognitive, and Clinical Neuroimaging Laboratory, Division of Experimental Medicine, Imperial College London, Hammersmith Hospital \\ Campus, London W12 0NN, United Kingdom, and ${ }^{2}$ Centre for Functional Magnetic Resonance Imaging of the Brain, University of Oxford, Oxford OX3 \\ 9DU, United Kingdom
}

The posterior cingulate cortex (PCC) is a central part of the default mode network (DMN) and part of the structural core of the brain. Although the PCC often shows consistent deactivation when attention is focused on external events, anatomical studies show that the region is not homogeneous, and electrophysiological recordings in nonhuman primates suggest that it is directly involved in some forms of attention. We report a functional magnetic resonance imaging study of an attentionally demanding task (either a zero- or two-back working memory task). Standard subtraction analysis within the PCC shows a relative deactivation as task difficulty increases. In contrast, a dual-regression functional connectivity analysis reveals a clear dissociation between ventral and dorsal parts of the PCC. As task difficulty increases, the ventral PCC shows reduced integration within the DMN and less anticorrelation with the cognitive control network (CCN) activated by the task. The dorsal PCC shows an opposite pattern, with increased DMN integration and more anticorrelation. At rest, the dorsal PCC also shows functional connectivity with both the DMN and attentional networks. As expected, these results provide evidence that the PCC is involved in supporting internally directed thought, as the region is more highly integrated with the DMN at low task demands. In contrast, the task-dependent increases in connectivity between the dorsal PCC and the CCN are consistent with a role for this region in modulating the dynamic interaction between these two networks controlling the efficient allocation of attention.

\section{Introduction}

The posterior cingulate cortex (PCC) is a central node within the structural core of the brain. It is highly anatomically connected (Hagmann et al., 2008), has a high baseline metabolic rate (Raichle et al., 2001), and is a prominent part of the default mode network (DMN) (Buckner et al., 2008). Despite its apparent importance, there is no clear consensus about its function.

Increased activity is observed within the PCC when individuals direct attention internally (Shannon and Buckner, 2004; Szpunar et al., 2007). Conversely, when attention is directed externally, the region deactivates (Singh and Fawcett, 2008). A failure to maintain deactivation during externally directed tasks is associated with attentional lapses (Weissman et al., 2006), which could represent a disruptive effect of higher DMN activity on behavioral control systems (Sonuga-Barke and Castellanos, 2007). However, greater functional integration within the DMN (Hampson et al., 2006) and greater anticorrelation with a coupled cognitive control network (CCN) (Kelly et al., 2008) are both associated with improved behavioral performance when atten-

\footnotetext{
Received 0ct. 27, 2010; accepted Nov. 17, 2010.

This work was supported by the Medical Research Council (United Kingdom) and Research Councils United Kingdom.

Correspondence should be addressed to Robert Leech, Computational, Cognitive, and Clinical Neuroimaging Laboratory, Division of Experimental Medicine, Imperial College London, Hammersmith Hospital Campus, Du Cane Road, London W12 0NN, UK. E-mail: r.leech@imperial.ac.uk.

DOI:10.1523/JNEUROSCI.5626-10.2011

Copyright $\odot 2011$ the authors $\quad 0270-6474 / 11 / 313217-08 \$ 15.00 / 0$
}

tion is directed externally. Electrophysiological recordings in nonhuman primates also show that single-cell firing within PCC predicts subsequent behavior (Hayden et al., 2008, 2009). These findings suggest an alternative hypothesis that the PCC is actively involved in controlling the focus of attention, possibly maintaining a vigilant attentional state (Gilbert et al., 2007).

It may be possible to reconcile these competing theories by recognizing that the PCC is functionally heterogeneous. Although parts of the PCC, retrosplenial cortex, and precuneus are sometimes treated as functionally homogenous within the DMN, subregions show different patterns of functional connectivity (Margulies et al., 2009). Distinct cytoarchitectonics within the PCC suggest separation into dorsal (anterior) and ventral (posterior) areas (Vogt et al., 2006). At rest, the ventral PCC is integrated with the DMN, whereas the dorsal PCC shows functional correlation both with the DMN and with brain regions typically involved in the control of externally directed behavior (Vincent et al., 2008; Margulies et al., 2009). The more widespread intrinsic functional connectivity of the dorsal PCC suggests this region may be an interface between the DMN and CCN; however, this potential role has not been explicitly tested using varying task demands.

Here, we provide evidence that the dorsal and ventral parts of the PCC have differential roles in attentional control. We first consider intrinsic connectivity at "rest" to investigate whether the PCC is functionally connected to neural attentional systems. Subsequently, we analyze network dynamics within the DMN, 
during performance of a working memory task with varying attentional demands. We test whether attentional load modulates functional connectivity within the PCC. The degree to which each voxel within the PCC is functionally associated with the rest of the network provides a measure of network integration. We test whether the general PCC deactivation observed with high working memory load is associated with distinct patterns of functional connectivity within subregions of the PCC. Specifically, we investigate whether subregions within the PCC show distinct interactions with the rest of the DMN and distinct anticorrelations with the $\mathrm{CCN}$ that reflect task difficulty.

\section{Materials and Methods \\ Participants}

Twelve subjects were recruited for the study (nine males; mean age, $28 \pm 5$ ). Subjects gave written consent. The experiment was approved by the Hammersmith and Queen Charlotte's and Chelsea Research ethics committee.

\section{Procedure}

Initially, subjects received a 10 min resting state scan. After this, subjects were scanned during four conditions: less attentionally demanding zeroback or more demanding two-back task, presented in either the visual (written digits from 0 to 9 ) or auditory (spoken digits from 0 to 9 ) modality. Each condition was presented as a separate 3.5 min scanning run, with 9 min of resting state acquisition in between. Additional resting state scans were collected flanking the task scans to investigate whether there were residual traces of task activation observable in subsequent rest (this is not the focus of the current study and will not be discussed further). Subjects attended to the stimuli either via headphones or visually on the screen. Stimuli were presented for $0.5 \mathrm{~s}$ for visual stimuli and approximately the same length for auditory stimuli with an interstimulus interval of $1.5 \mathrm{~s}$. For the zero-back tasks, subjects responded to the target stimuli (the digit 0 ) with a button press. For the two-back task, participants detected a repetition of the digit occurring two trials before. Target trials occurred in $20 \%$ of the trials. Subjects were instructed to respond as quickly and as accurately as possible. To maximize design efficiency, the tasks had a blocked design: $32 \mathrm{~s}$ of task followed by $10 \mathrm{~s}$ of fixation at a cross. There were five blocks in each run. The order of presentation of the four different conditions was counterbalanced across subjects. Accuracy data for the zero-back condition are from additional testing of a subset of the original subjects outside the scanner because of a technical fault leading to a failure of accuracy recordings during the zero-back condition.

\section{Image acquisition}

For functional magnetic resonance imaging (fMRI), T2* ${ }^{*}$ weighted gradient echo planar images were collected with whole-brain coverage, with the following parameters: repetition time, $2 \mathrm{~s}$; echo time, $30 \mathrm{~ms} ; \alpha=90^{\circ}$; 32 axial slices; slice thickness, $3.25 \mathrm{~mm}$; interslice gap of $0.75 \mathrm{~mm}$; acquisition in ascending order (resolution: 2.19, 2.19, $4.0 \mathrm{~mm}$ ). Quadratic shim gradients were applied to correct for inhomogeneities in the magnetic field. A high-resolution $1 \mathrm{~mm}^{3}$ T1-weighted structural image was also obtained for each subjects. Paradigms were programmed using Matlab Psychophysics Toolbox (Psychtoolbox-3; www.psychtoolbox.org) and stimuli presented through an IFIS-SA system (In Vivo Corporation). Responses were recorded through a fiber optic response box (Nordicneurolab), interfaced with the stimulus presentation PC running Matlab. Sounds were presented using ear-defending headphones (MR Confon).

\section{Functional MRI analysis of task activation}

A high-level description of how both the task and rest fMRI data were analyzed is presented in Figure 1. Whole-brain fMRI data from the $\mathrm{N}$-back task were analyzed with standard random-effects general linear models using tools from the FSL library (FEAT, version 5.98) (Smith et al., 2004). Image preprocessing involved realignment of echo planar imaging (EPI) images to remove the effects of motion between scans, spatial smoothing using a $6 \mathrm{~mm}$ full-width half-maximum Gaussian kernel, prewhitening using FILM (FMRIB's improved linear model), and temporal high-pass filtering using a cutoff frequency of $1 / 50 \mathrm{~Hz}$ to correct for baseline drifts in the signal. FMRIB's linear image registration tool was used to register EPI functional datasets into standard Montreal Neurological Institute (MNI) space using the participant's individual highresolution anatomical images. fMRI data were analyzed using voxelwise time series analysis within the framework of the general linear model (GLM). To this end, a design matrix was generated with a synthetic hemodynamic response function and its first temporal derivative. Task and rest blocks were modeled in the design matrix for each run. A factorial ANOVA investigated effects of task difficulty, modality, and interactions across the 12 subjects using FMRIB's local analysis of mixed effects (Beckmann et al., 2003). Final statistical images were thresholded using Gaussian random field-based cluster inference with a height threshold of $Z>2.3$ and a cluster significance threshold of $p<0.05$.

\section{Analysis of functional connectivity}

Resting state data. The data from the resting state scans of the 12 subjects were combined and analyzed using group temporal concatenation independent component analysis (ICA) using MELODIC (3.10), part of FSL (Beckmann et al., 2005). ICA and group-ICA extract a variety of structured signals that can exist simultaneously in the data. As such, it is able to separate signals of interest (e.g., patterns related to intrinsic functional connectivity) from effects of head motion and physiological noise. Equally, ICA can reveal distinct but partially spatially overlapping networks, corresponding to functionally different activation time courses intermixed within a single voxel. Therefore, ICA can discover functional networks that would be missed in standard subtraction activation analyses. Temporal concatenation ICA is appropriate for data such as restingstate fMRI where there is no underlying time course common across subjects. The group ICA was used to define 30 independent components. These components were thresholded to only include voxels with $p>0.5$ probability of activation based on a Gaussian/Gamma mixture model (Beckmann and Smith, 2004). Subsequently, three canonical restingstate independent components were identified using a template matching procedure (Garrity et al., 2007) with reference to the original resting 


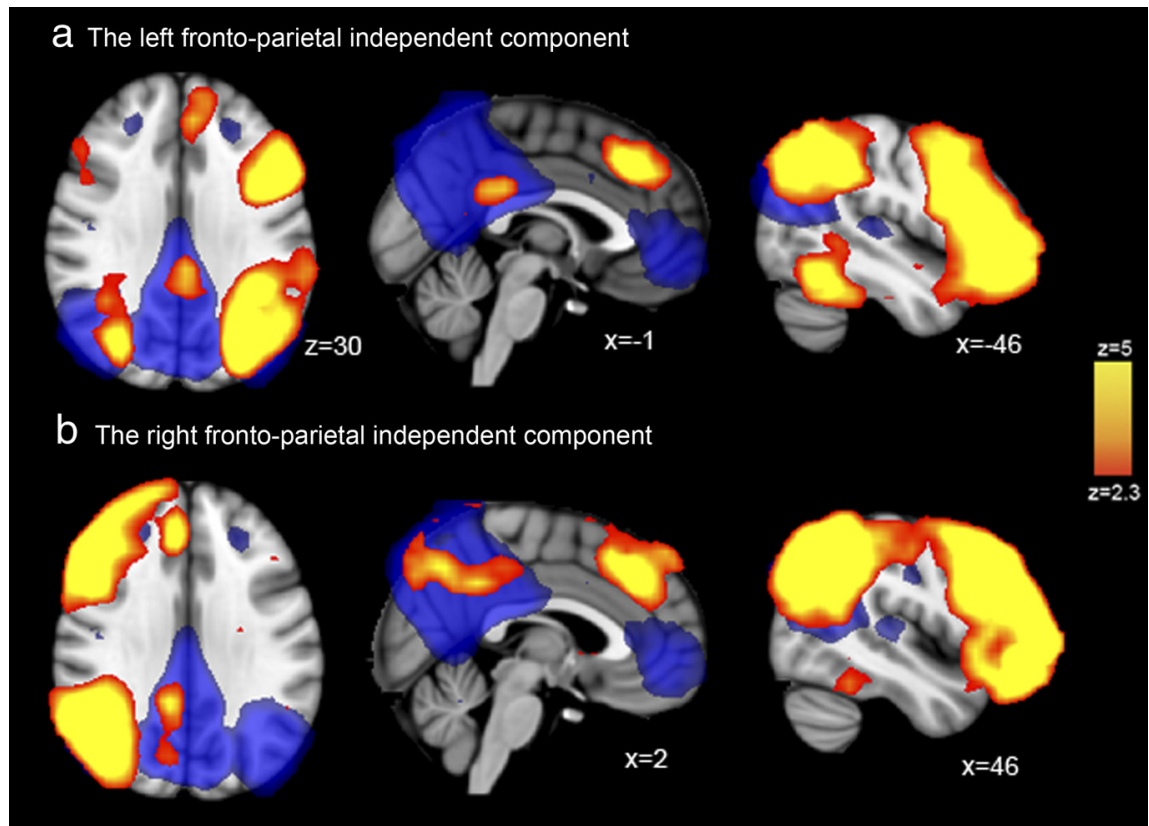

Figure 2. Whole-brain results from a data-driven ICA analysis of resting-state fMRI data. The yellow regions in $\boldsymbol{a}$ and $\boldsymbol{b}$ are two distinct lateralized frontoparietal independent components derived from the whole population, typical of resting-state fMRI. Of note is that both of the frontoparietal components also contain dorsal PCC regions. To illustrate the relationship between these connected parts of the PCC and the DMN, the blue underlay shows the typical DMN, which is frequently observed with resting-state fMRI. MNI coordinates are shown for each slice.
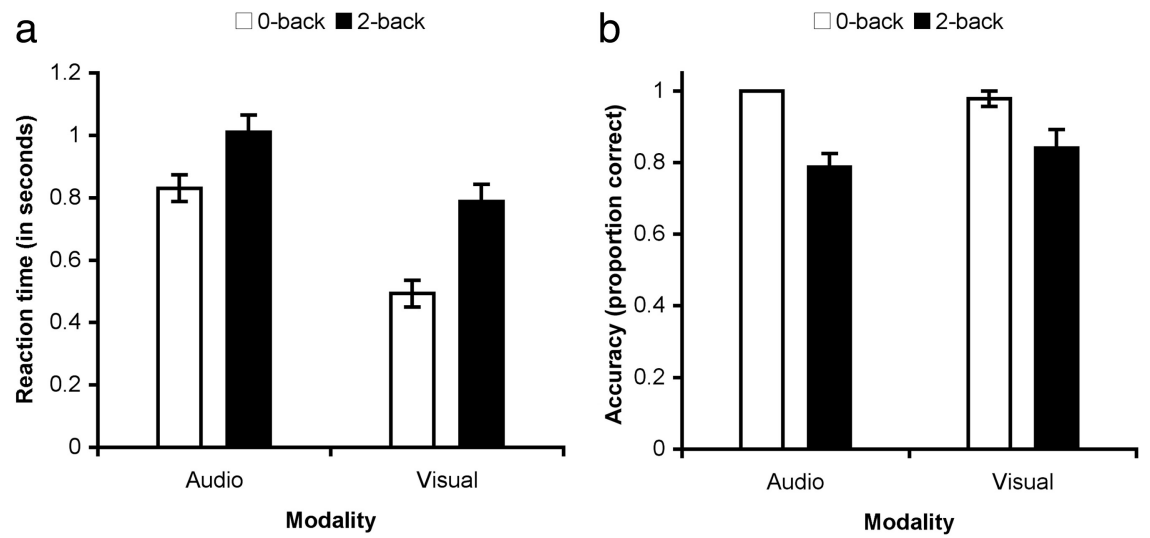

Figure 3. Behavioral results for the $N$-back task. $a$, Group mean and $S E$ of reaction time across participants for correct responses. $\boldsymbol{b}$, Group mean and SE of the proportion of correct responses.

state networks delineated in previous work using ICA (Beckmann et al., 2005). The default mode network and a left and a right lateralized frontoparietal networks from the study by Beckmann et al. (2005) were chosen as templates and correlated with each independent component identified at rest in the current study. The component with the highest Pearson $r$ statistic was chosen as the network resembling that identified in multiple previous studies (Beckmann et al., 2005; Smith et al., 2009).

Task data. A group-based independent component analysis was used with dual regression to study voxelwise functional connectivity under different task conditions within different parts of the PCC (Filippini et al., 2009; Zuo et al., 2010). This approach has a number of advantages over the use of seed-voxel-based approaches by covarying out nuisance variables and by avoiding the need to a priori choose regions of interest (Zuo et al., 2010) and has previously been used to probe for behavioral and pathology-related differences (Filippini et al., 2009). Tensor-ICA (Beckmann and Smith, 2005) as implemented in the MELODIC software was first used to define 25 reference network maps common across all four conditions (zero- or two-back, visual or auditory modality).
Tensor-ICA is appropriate for data where there is a common time course across subjects such as task-based fMRI. Subsequently, network dynamics across the four conditions were compared as follows: (1) the reference networks were used to derive individual time courses for each component for each subject by means of linearly regressing each subjects' data against the spatial maps derived from tensor-ICA; (2) these subject- and network-specific time courses were then normalized to unit SD and re-regressed onto each subject's data resulting in a subject-specific spatial map of network coherence for each initial reference independent component map. The resulting spatial maps for each subject were tested for voxelwise between-subject differences using nonparametric permutation testing of a factorial ANOVA including task difficulty, modality, and interactions. Five thousand random permutations were calculated to create the null distribution for assessing the test statistics. Random permutation-based statistics can be conservative, a problem confounded by the multiple-comparison problem present for whole-brain fMRI analysis. To avoid this limitation, a theoretically chosen anatomical region of interest encompassing the PCC was defined based on combining thresholded (at a probability of $>20 \%$ ) posterior cingulate and precuneus probabilistic regions of interest from the Harvard Atlas anatomical atlas. Results were cluster corrected (using a nominal $t$ value of 2 ) to control for multiple comparisons.

Given the theoretical question, not all independent component spatial maps were considered. Instead, the analysis was focused on a single component capturing the DMN as well as its counterpoint, the CCN or task-positive network (involving frontoparietal systems that reliably activate on task). As such, the analysis tests whether voxels within the PCC increase or decrease in how functionally connected they are with the DMN and CCN as task demands change.

\section{Results}

\section{The PCC at rest}

A group independent components analysis was performed on fMRI data acquired from 12 subjects at rest. The analysis qualitatively replicated previous group-ICA analyses, revealing a canonical DMN (Fig. 2, blue), with ventromedial prefrontal, medial parietal (including large parts of the PCC and precuneus), and lateral parietal regions highly functionally interconnected at rest. Two other left and right lateralized frontoparietal networks independent components were identified, which included the dorsal PCC. These components overlap considerably with networks that have been identified in attentionally demanding cognitive tasks (Sharp et al., 2010) and that appear likely to be involved in different facets of cognitive control (Smith et al., 2009). This analysis suggests that, although the DMN, including the PCC, is highly interconnected as a whole, the dorsal PCC is also functionally connected to regions involved in cognitive control. Although this result is informative and is in keeping with previous work using seed-voxel-based approaches to functional connectivity (Vincent et al., 2008; Margulies et al., 2009), to fully 


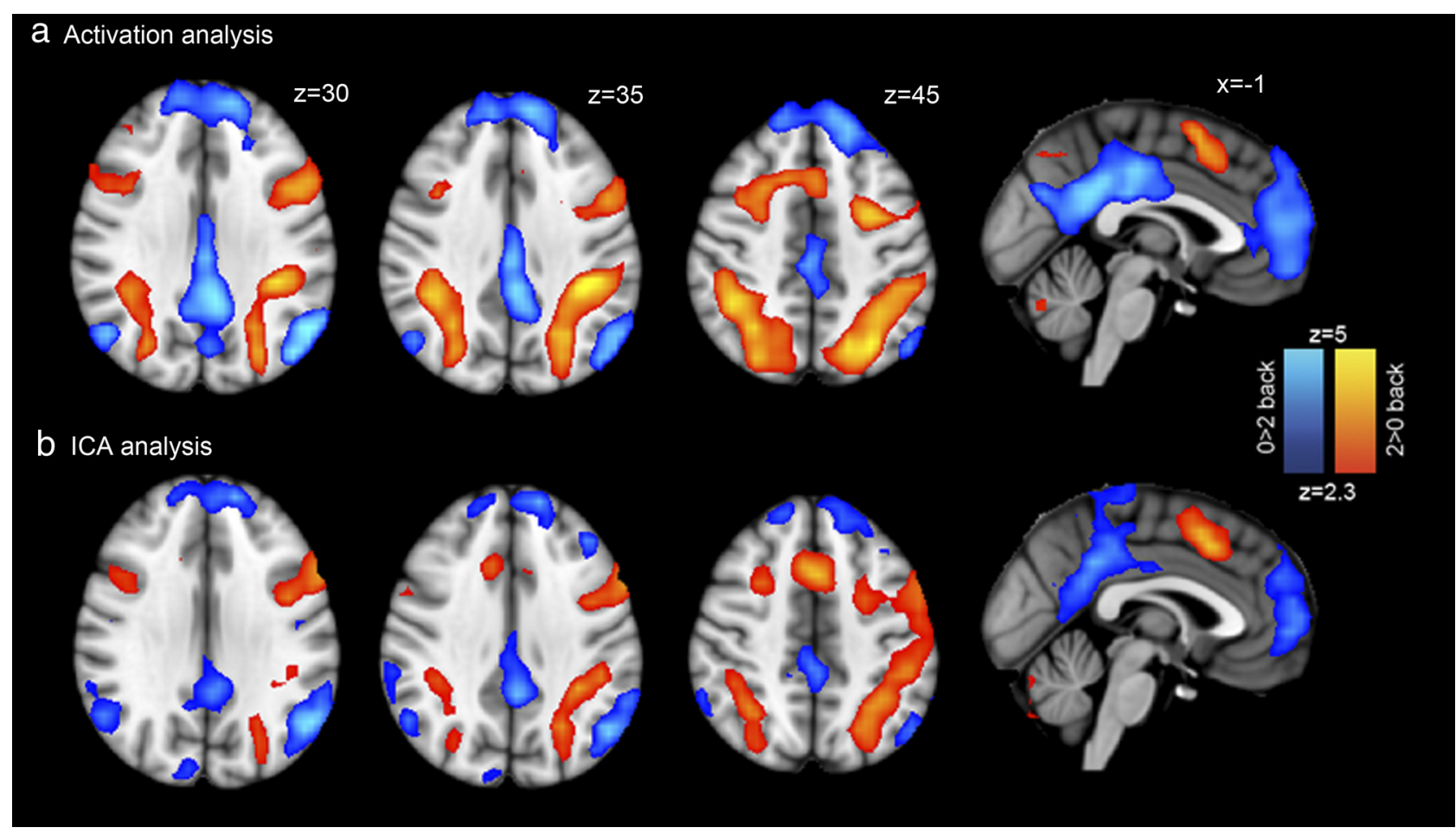

Figure 4. Cognitive control and default mode networks associated with $N$-back performance. $\boldsymbol{a}$, Whole-brain results from general linear model analysis. Red-yellow regions show increase in activation with task difficulty (i.e., two-back $>$ zero-back) within a frontoparietal executive network (cognitive control network). Blue-light blue regions show regions that deactivate with task difficulty (zero-back $>$ two-back) within the DMN. Results are cluster corrected at $p<0.05$. $\boldsymbol{b}$, Whole-brain results from the data-driven ICA analysis. One component closely reflects the GLM analysis, showing activation of the frontoparietal executive network and deactivation within the DMN. Results are thresholded at a $p>0.5$ level under an alternative hypothesis test based on a Gaussian/Gamma mixture model fitted to the intensity histogram of the component.

understand the functional role of the PCC requires investigation of how its activation and functional connectivity is modulated by task demands.

\section{The PCC during task}

Behavioral results for $\mathrm{N}$-back task

To investigate the relationship between different parts of the PCC and an executive network engaged during attentionally demanding task performance, we used an $\mathrm{N}$-back task. Previous studies have shown that a frontoparietal executive system is activated more by the two- than zero-back version of the task and that the two-back task is associated with a relative deactivation within the default mode network (Braver et al., 1997; Esposito et al., 2006). As expected, accuracy was higher and reaction time faster in the zero-back task compared with the two-back task (Fig. 3). This was true in both the visual and auditory modality. Reaction time measures for the visual modality cannot be directly compared with the auditory modality given the temporal difference in presentation of an auditory and visual stimulus. However, accuracy (mean proportion of correct responses) was similar in both modalities.

Coupled activation and deactivation within the cognitive control network and the DMN

We first investigated activation changes in the $N$-back task using a GLM-based analysis, explicitly modeling the timings of $N$-back blocks. The attentionally demanding two-back condition was associated with greater activation than the zero-back within a frontoparietal network frequently identified in this type of executive task, which included regions involved in cognitive control (Fig. $4 a)$. Peaks of activation were seen within lateral and superior parietal regions, as well as medial and lateral frontal regions (Ta-
Table 1. Activation peaks from within significant clusters $(p<0.05$ cluster corrected) for contrast of zero- $>$ two-back and two- $>$ zero-back working memory tasks

\begin{tabular}{llrrr}
\hline & $Z$ & & \\
\hline & & & & \\
\hline Peak activation for zero- $>$ two-back (DMN) & & & \\
$\quad$ Ventral medial prefrontal cortex & 5.55 & 6 & 70 & 14 \\
Superior frontal gyrus & 4.92 & 8 & 50 & 38 \\
$\quad$ Ventral posterior cingulate & 5.23 & -6 & -48 & 26 \\
Dorsal posterior cingulate & 5.11 & 0 & -24 & 38 \\
Precuneus cortex & 4.83 & -8 & -60 & 14 \\
Ventral posterior cingulate & 4.71 & 2 & -46 & 28 \\
Left inferior parietal lobe & 5.73 & -56 & -66 & 24 \\
Right inferior parietal lobe & 4.82 & 50 & -62 & 30 \\
Peak activation for two- $>$ zero-back (CNN) & & & & \\
Right orbital frontal cortex & 6.18 & 24 & 10 & -10 \\
Right putamen & 6.18 & 22 & 14 & -6 \\
Left putamen & 5.99 & -20 & 10 & -10 \\
Left orbital frontal cortex & 5.81 & -16 & 16 & -12 \\
Left inferior frontal gyrus, pars opercularis & 5.8 & -38 & 10 & 26 \\
Left middle frontal gyrus & 5.61 & -36 & 2 & 52 \\
Right inferior parietal lobe, angular gyrus & 6.44 & 20 & -68 & 60 \\
Left inferior parietal lobe, angular gyrus & 5.88 & -24 & -70 & 54 \\
Left supramarginal gyrus & 5.56 & -44 & -32 & 34 \\
Right cerebellum VI & 4.28 & 24 & -64 & -32 \\
Right cerebellum crus I & 4.09 & 46 & -52 & -38 \\
Left cerebellum crus I & 3.76 & -22 & -66 & -36 \\
\hline
\end{tabular}

ble 1). Given the overlap between this network and that found in many previous fMRI tasks that involve externally directed, cognitively mediated control of behavior, from here on we will refer to the network as the CCN. The same contrast showed deactivation on the two-back task within what is generally defined as the 


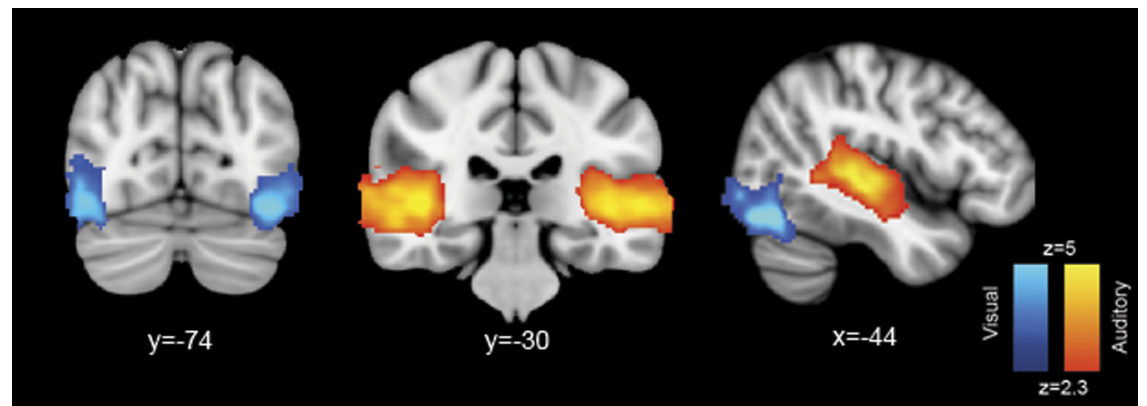

Figure 5. Activation differences between the auditory and visual versions of the $N$-back tasks. Increased auditory $>$ visual activation is in red, and the reverse contrast is in blue (results are cluster corrected, $p<0.05$ ).

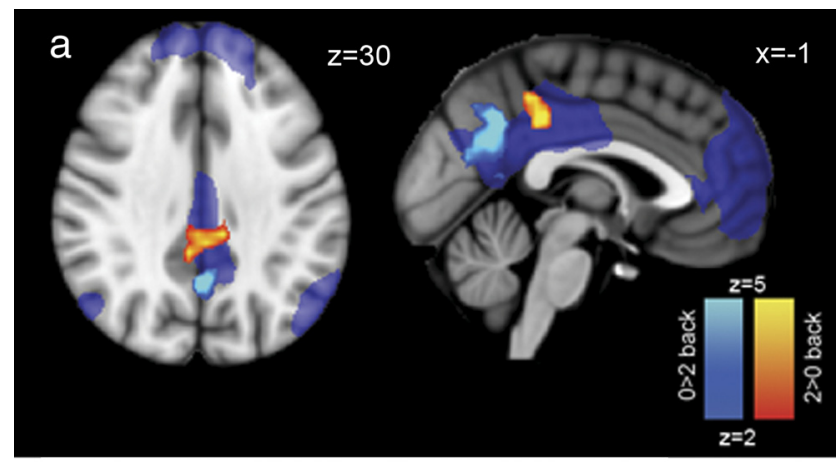

b 口 Zero-back $\square$ Two-back

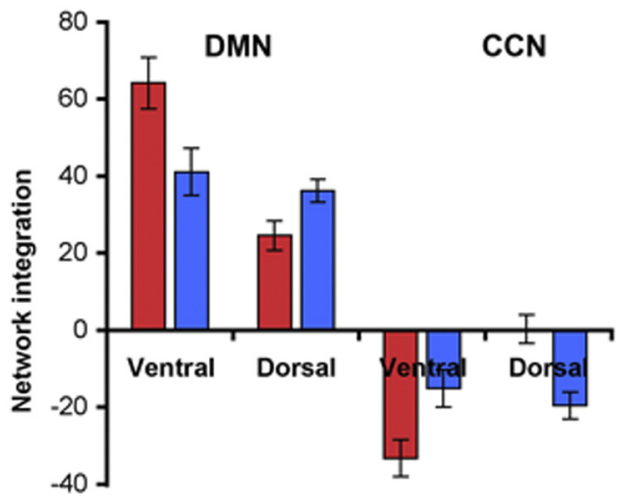

Figure 6. Distinct connectivity within the dorsal and ventral PCC. $\boldsymbol{a}$, Increasing integration with task difficulty in the dorsal PCC (i.e., two-back $>$ zero-back) is shown in red-yellow. Decreases in integration with task difficulty in the ventral PCC (i.e., zero-back $>$ two-back) are shown in dark-light blue. The areas showing relative deactivation for two-back $>$ zero-back are shown as a transparent blue (i.e., the DMN revealed in Fig. $3 a$ ). Results are cluster corrected at $p<0.05 . \boldsymbol{b}$, Integration values for the peak voxels (i.e., lowest $p$ value) of the dorsal and the ventral $P C C$, shown combined for auditory and visual presentation. Integration values are the regression $\beta$ coefficients resulting from the dual regression at each peak voxel. Integration is calculated separately for the DMN (on the left) and the CCN (on the right). The peak ventral PCC voxel was located at MNI 2, $-58,28$. The peak dorsal PCC voxel was at MNI 2, $-34,40$.

$\mathrm{DMN}$, with peaks within the PCC/precuneus, lateral parietal lobes, and ventromedial prefrontal cortex (PFC). Deactivation of the medial posterior portion of the DMN with task was extensive around the posterior part of the corpus callosum, including both the ventral and dorsal PCC. The contrast of different modalities (auditory vs visual stimulus presentation) also produced expected results, with superior temporal regions increasing in activation for auditory stimuli and higher-order visual regions increasing in activation for visual stimuli (Fig. 5). There was no significant interaction between modality and task difficulty.
Similar default and executive networks are identified using independent

component analysis

As the first stage of our comparison of network dynamics, we used ICA to identify regions showing correlated activity during task performance (Beckmann et al., 2005). This approach typically identifies a number of characteristic cognitive networks (Smith et al., 2009), and in our analysis one component showed a high degree of similarity to the results of the GLM analysis (Fig. 4b). In this component, strong functional connectivity was observed in the frontoparietal network activated by two-back performance. An anticorrelated network mostly overlapping with the DMN was also present in the same component. The correlation between the results of the GLM and ICA analyses (including both DMN and CCN) was high (whole-brain voxelwise correlation, $r=0.69$ ). This suggests equivalence of the underlying neural networks estimated by the GLM and ICA approaches. The ICA DMN/CCN is used from here on in the dual-regression analysis; this contains the same broad pattern of regions as the GLM analysis but also allows us to covary out other components (e.g., movement and physiological noise components) as well controlling for the influence of other brain networks.

\section{Dorsal and ventral PCC show distinct connectivity during attentional control}

We investigated the functional connectivity of the DMN using a dual-regression analysis. This builds on the output of the ICA analysis and provides a voxel-based measure of network integration for each individual (see Materials and Methods) (Beckmann et al., 2005; Filippini et al., 2009; Zuo et al., 2010). A voxel with a high regression value between its local brain activity and the average brain activity for the whole network has a high integration value. A negative value for integration signifies anticorrelation between the activation time course at that voxel and the overall network-characteristic time course. The method allows regionally specific patterns of functional connectivity to be identified in a data-driven way.

We first examined integration within different parts of the PCC/precuneus and the whole of the correlated and anticorrelated set of brain regions identified in the ICA component (i.e., the DMN and the CCN). Our analysis identified two spatially separable clusters of voxels showing either increasing or decreasing functional connectivity with the regions identified in the ICA component. Distinct patterns of functional connectivity were seen in the dorsal and ventral PCC during the high and low task difficulty conditions (Fig. 6a). The ventral PCC showed a reduction of integration with the overall ICA component. In contrast, the dorsal PCC showed the reverse pattern, becoming more integrated with increasing task difficulty. These differential patterns of network integration fell mostly within the large area extending across the PCC and precuneus that showed significant deactivation on the two-back task. Therefore, relative changes in regional activation and functional connectivity in the dorsal PCC disassociate, whereas both activation and connectivity decline with task difficulty in the ventral PCC.

The single ICA component we analyzed included regions that encompassed both the DMN and CCN. For a given independent component, individual voxels have either a positive or negative weight, reflecting correlation or anticorrelation with the time course of the overall component. Voxels within the DMN and 
within the $\mathrm{CCN}$ were extracted in the same independent component because they showed high but opposite functional connectivity with the overall component. Because both DMN and CCN were included in the same component, the distinct patterns of integration we observed within the dorsal and ventral PCC could have arisen as a result of changes in correlation either to the $\mathrm{DMN}$, or in anticorrelation to the CCN, or a combination of the two. To distinguish between these possibilities, two additional dual-regression analyses were performed, separating the DMN and CCN into different components. A dual regression was then run with either the DMN included and no CCN or vice versa but not both. Thus, any task-modulated differences in integration found for the subsequent analyses will relate only to change in voxels within the DMN or within the CCN. This provides a measure akin to correlation of the parts of the PCC with the DMN or anticorrelation with the CCN. Measures of integration were assessed at the peak voxel of the ventral and dorsal parts of the PCC. In high task difficulty, the ventral PCC showed both reduced integration with the DMN and reduced anticorrelation with the CCN (Fig. 6b). In contrast, the dorsal PCC showed the opposite pattern, increasing both its correlation with the DMN and its anticorrelation with the CCN. Thus, relative deactivation within large parts of PCC is associated with distinct changes in integration in the dorsal and ventral PCC with task demand, the result of symmetrical changes in correlation and anticorrelation to the rest of the DMN and coupled CCN.

\section{Discussion}

The PCC is a hub within the brain, connecting networks that function together to support complex behavior (Hagmann et al., 2008). Although the region is a core part of the DMN that deactivates on many cognitive tasks, it shows rapid and highly reactive changes in brain activation that depend on cognitive demands (Singh and Fawcett, 2008; Pyka et al., 2009). Here, we demonstrate that at "rest" the dorsal PCC is functionally connected to distinct distributed networks thought to be closely involved in the control of behavior, as well as being highly integrated within the DMN (Fig. 7). Furthermore, performance of an attentionally demanding task modulates functional connectivity of the dorsal and ventral parts of the PCC in opposite directions, despite both regions showing similar reductions in overall activation. As task difficulty increases, the ventral PCC becomes less integrated with the DMN and becomes less anticorrelated with the task-positive CCN. In contrast, the dorsal PCC becomes more integrated with the DMN and shows greater CCN anticorrelation. This demonstrates that the dorsal and ventral parts of the PCC are functionally distinct and suggests that the PCC may serve as an interface between distinct distributed brain networks involved in the control of internally or externally directed cognition.

A failure to maintain deactivation with the PCC is associated with attentional lapses (Weissman et al., 2006), and individual differences in functional connectivity between the PCC and the ventromedial PFC are known to correlate with improved performance on a working memory task (Hampson et al., 2006). In addition, the degree of anticorrelation between the DMN and an executive network has also been shown to correlate with individual differences in behavior, such that greater anticorrelation is associated with a more consistent behavioral performance (Kelly et al., 2008). These previous findings demonstrate that reduced activation within the DMN does not necessarily signify a disengagement of the DMN and that the relationship between coupled but anticorrelated networks may be important for the control of efficient goaldirected behavior. We extend these findings by demonstrating

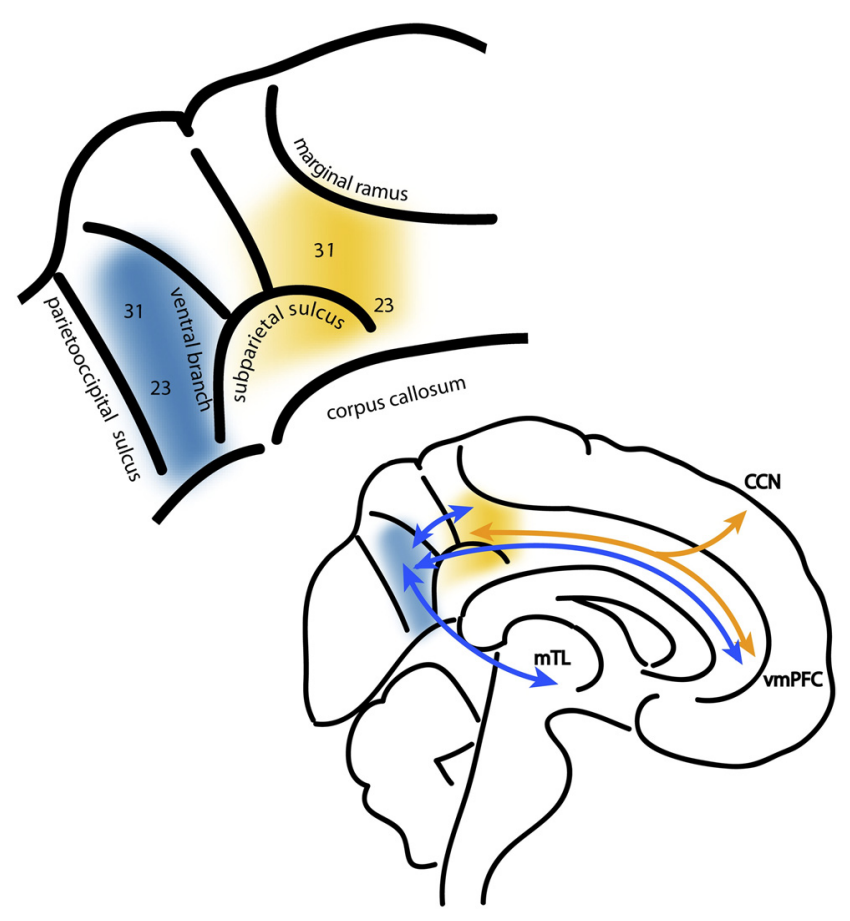

Figure 7. Top, The proposed functional subdivisions of PCC. The dorsal PCC is shown in warm colors and the ventral PCC in cold colors. The ventral branch of the subparietal sulcus anatomically separates the two functional regions (Vogt et al., 2006). Brodmann cytoarchitectonic regions 23 and 31 are labeled. Bottom, A schematic of the functional connectivity from the PCC subdivisions. The ventral and dorsal PCC are strongly interconnected and project to other regions of the DMN, including the ventromedial prefrontal cortex (vmPFC), and inferior parietal lobe (data not shown). The dorsal PCC shows stronger functional connectivity to "task-positive" regions within the cognitive control network, and we propose that the ventral PCC is more strongly functionally connected to medial temporal lobe (mTL) structures including the hippocampus.

complementary patterns of functional connectivity within the dorsal and ventral PCC as attentional load increases. Efficient behavior was associated with both deactivation within the dorsal PCC and increased functional coupling to the CCN. This suggests that coupling of the two systems may be mediated in part through the dorsal PCC.

The ventral PCC, however, may be fulfilling a functional role more traditionally associated with the DMN. There is considerable evidence that the DMN has a role in supporting internally directed thought (Shannon and Buckner, 2004; Wheeler and Buckner, 2004; Vincent et al., 2006; Buckner et al., 2008), and the changes in ventral PCC connectivity we observe are consistent with this proposal. The ventral PCC is activated during tasks that require an internal focus of attention, such as autobiographical memory retrieval (Svoboda et al., 2006) and envisioning the future (Schacter et al., 2007). Furthermore, lesions around the retrosplenial cortex and PCC produce an amnestic state (Valenstein et al., 1987). Viewed in this context, the relative deactivation of the PCC we observe during high task difficulty might be interpreted as reflecting a suppression of internally directed thought, in favor of an external focus of attention. The reduction of ventral PCC integration would be consistent with this idea. The contrast between the task-dependent changes in functional connectivity observed in the dorsal and ventral PCC provides additional evidence for functional heterogeneity within the PCC. These results demonstrate that subregions within the PCC show a flexible recruitment in different modes of cognitive function, which is dependent on current task demands and is expressed in changing patterns of functional connectivity. 
The functional differences between dorsal and ventral parts of the PCC are reflected in structural differences. The PCC (area 23) has previously been separated on the basis of postmortem histology into dorsal (more anterior) and ventral (more posterior) regions (Vogt et al., 2006). These subregions have distinct cytoarchitectonics, different patterns of structural connectivity, and varying resting state functional connectivity (Vogt and Laureys, 2005; Vogt et al., 2006; Margulies et al., 2009). The dorsal PCC has previously been shown to have greater prefrontal functional connectivity at rest, particularly with the medial PFC (Vogt et al., 2006; Margulies et al., 2009), whereas the ventral PCC has greater temporal lobe functional connectivity (Choi et al., 2006). These differences are preserved across species (Margulies et al., 2009) and are consistent with direct studies of PCC connectivity in nonhuman primates (Morecraft et al., 2004). The dorsal PCC has also been shown to exhibit resting-state functional connectivity both with the DMN and with frontoparietal regions involved in the executive control of behavior (Vincent et al., 2008; Margulies et al., 2009). We report a qualitatively similar result in restingstate data using an ICA approach.

Our results highlight the importance of distinguishing between changes in the magnitude of activation within the PCC and changes in its functional connectivity with other brain regions. As expected, we found a general deactivation of the PCC during task performance, but in the dorsal PCC this was accompanied by increased integration of the dorsal PCC into the DMN and reduced integration (increased anticorrelation) with the CCN. A recent computational model of neural dynamics provides a potential mechanism that could explain how a local increase in functional integration within the PCC could influence behavior even though it is accompanied by an overall decrease in activation (Schacter et al., 2007). Deco et al. (2009) show how antiphase network oscillations (similar to the anticorrelations reported here and previously between the DMN and CCN) can emerge, without the need for top-down inhibition, as a result of interactions between the DMN and a coupled "executive" network. The authors propose that this oscillation may be behaviorally relevant in making the network more efficient and responsive to a dynamic environment. The magnitude of anticorrelation is in part a function of local connectivity within each network, so greater integration of the PCC with the DMN would result in greater anticorrelation between the two networks. We show that increasing task difficulty is associated with both increased integration of the PCC within the DMN and greater anticorrelation with a coupled executive network activated by the task. As greater anticorrelation between the DMN and a similar cognitive control network is associated with more efficient behavior (Kelly et al., 2008), this provides a mechanism by which task-related changes in PCC connectivity might influence behavior through the "tuning" of anticorrelations in behaviorally relevant networks.

In this study, the dorsal and ventral PCC show distinct patterns of functional connectivity, both at rest and during performance of an attentionally demanding task. The dorsal PCC shows functional connectivity with both the DMN and the CCN, two competing networks involved in either internally or externally directed thought. Efficient behavior requires a regulated balance between these two modes of cognitive operation, and the pattern of connectivity shown by the dorsal PCC is consistent with a role interfacing between the two systems. We propose that the ventral PCC is directly involved in internally directed thought, in which the demands for externally directed attention are low, whereas the dorsal PCC is actively involved in managing attention di- rected externally, in keeping with an increase in functional integration as task difficulty increases.

\section{References}

Beckmann CF, Smith SM (2004) Probabilistic independent component analysis for functional magnetic resonance imaging. IEEE Trans Med Imaging 23:137-152.

Beckmann CF, Smith SM (2005) Tensorial extensions of independent component analysis for group FMRI data analysis. Neuroimage 25:294-311.

Beckmann CF, Jenkinson M, Smith SM (2003) General multilevel linear modeling for group analysis in FMRI. Neuroimage 20:1052-1063.

Beckmann CF, DeLuca M, Devlin JT, Smith SM (2005) Investigations into resting-state connectivity using independent component analysis. Philos Trans R Soc Lond B Biol Sci 360:1001-1013.

Braver TS, Cohen JD, Nystrom LE, Jonides J, Smith EE, Noll DC (1997) A parametric study of prefrontal cortex involvement in human working memory. Neuroimage 5:49-62.

Buckner RL, Andrews-Hanna JR, Schacter DL (2008) The brain's default network: anatomy, function, and relevance to disease. Ann N Y Acad Sci 1124:1-38.

Choi HJ, Zilles K, Mohlberg H, Schleicher A, Fink GR, Armstrong E, Amunts K (2006) Cytoarchitectonic identification and probabilistic mapping of two distinct areas within the anterior ventral bank of the human intraparietal sulcus. J Comp Neurol 495:53-69.

Deco G, Jirsa V, McIntosh AR, Sporns O, Kötter R (2009) Key role of coupling, delay, and noise in resting brain fluctuations. Proc Natl Acad Sci U S A 106:10302-10307.

Esposito F, Bertolino A, Scarabino T, Latorre V, Blasi G, Popolizio T, Tedeschi G, Cirillo S, Goebel R, Di Salle F (2006) Independent component model of the default-mode brain function: assessing the impact of active thinking. Brain Res Bull 70:263-269.

Filippini N, MacIntosh BJ, Hough MG, Goodwin GM, Frisoni GB, Smith SM, Matthews PM, Beckmann CF, Mackay CE (2009) Distinct patterns of brain activity in young carriers of the APOE-epsilon4 allele. Proc Natl Acad Sci U S A 106:7209-7214.

Garrity AG, Pearlson GD, McKiernan K, Lloyd D, Kiehl KA, Calhoun VD (2007) Aberrant "default mode" functional connectivity in schizophrenia. Am J Psychiatry 164:450-457.

Gilbert SJ, Dumontheil I, Simons JS, Frith CD, Burgess PW (2007) Comment on "Wandering minds: the default network and stimulusindependent thought." Science 317:43; author reply 43.

Hagmann P, Cammoun L, Gigandet X, Meuli R, Honey CJ, Wedeen VJ, Sporns O (2008) Mapping the structural core of human cerebral cortex. PLoS Biol 6:e159.

Hampson M, Driesen NR, Skudlarski P, Gore JC, Constable RT (2006) Brain connectivity related to working memory performance. J Neurosci 26:13338-13343.

Hayden BY, Nair AC, McCoy AN, Platt ML (2008) Posterior cingulate cortex mediates outcome-contingent allocation of behavior. Neuron 60:19-25.

Hayden BY, Smith DV, Platt ML (2009) Electrophysiological correlates of default-mode processing in macaque posterior cingulate cortex. Proc Natl Acad Sci U S A 106:5948-5953.

Kelly AM, Uddin LQ, Biswal BB, Castellanos FX, Milham MP (2008) Competition between functional brain networks mediates behavioral variability. Neuroimage 39:527-537.

Margulies DS, Vincent JL, Kelly C, Lohmann G, Uddin LQ, Biswal BB, Villringer A, Castellanos FX, Milham MP, Petrides M (2009) Precuneus shares intrinsic functional architecture in humans and monkeys. Proc Natl Acad Sci U S A 106:20069-20074.

Morecraft RJ, Cipolloni PB, Stilwell-Morecraft KS, Gedney MT, Pandya DN (2004) Cytoarchitecture and cortical connections of the posterior cingulate and adjacent somatosensory fields in the rhesus monkey. J Comp Neurol 469:37-69.

Pyka M, Beckmann CF, Schöning S, Hauke S, Heider D, Kugel H, Arolt V, Konrad C (2009) Impact of working memory load on FMRI resting state pattern in subsequent resting phases. PLoS One 4:e7198.

Raichle ME, MacLeod AM, Snyder AZ, Powers WJ, Gusnard DA, Shulman GL (2001) A default mode of brain function. Proc Natl Acad Sci U S A 98:676-682.

Schacter DL, Addis DR, Buckner RL (2007) Remembering the past to imagine the future: the prospective brain. Nat Rev Neurosci 8:657-661. 
Shannon BJ, Buckner RL (2004) Functional-anatomic correlates of memory retrieval that suggest nontraditional processing roles for multiple distinct regions within posterior parietal cortex. J Neurosci 24:10084-10092.

Sharp DJ, Bonnelle V, De Boissezon X, Beckmann CF, James SG, Patel MC, Mehta MA (2010) Distinct frontal systems for response inhibition, attentional capture and error processing. Proc Natl Acad Sci U S A 107:6106-6111.

Singh KD, Fawcett IP (2008) Transient and linearly graded deactivation of the human default-mode network by a visual detection task. Neuroimage 41:100-112.

Smith SM, Jenkinson M, Woolrich MW, Beckmann CF, Behrens TE, Johansen-Berg H, Bannister PR, De Luca M, Drobnjak I, Flitney DE, Niazy RK, Saunders J, Vickers J, Zhang Y, De Stefano N, Brady JM, Matthews PM (2004) Advances in functional and structural MR image analysis and implementation as FSL. Neuroimage 23 [Suppl 1]:S208-S219.

Smith SM, Fox PT, Miller KL, Glahn DC, Fox PM, Mackay CE, Filippini N, Watkins KE, Toro R, Laird AR, Beckmann CF (2009) Correspondence of the brain's functional architecture during activation and rest. Proc Natl Acad Sci U S A 106:13040-13045.

Sonuga-Barke EJ, Castellanos FX (2007) Spontaneous attentional fluctuations in impaired states and pathological conditions: a neurobiological hypothesis. Neurosci Biobehav Rev 31:977-986.

Svoboda E, McKinnon MC, Levine B (2006) The functional neuroanatomy of autobiographical memory: a meta-analysis. Neuropsychologia 44:2189-2208.

Szpunar KK, Watson JM, McDermott KB (2007) Neural substrates of envisioning the future. Proc Natl Acad Sci U S A 104:642-647.

Valenstein E, Bowers D, Verfaellie M, Heilman KM, Day A, Watson RT (1987) Retrosplenial amnesia. Brain 110:1631-1646.

Vincent JL, Snyder AZ, Fox MD, Shannon BJ, Andrews JR, Raichle ME, Buckner RL (2006) Coherent spontaneous activity identifies a hippocampal-parietal memory network. J Neurophysiol 96:3517-3531.

Vincent JL, Kahn I, Snyder AZ, Raichle ME, Buckner RL (2008) Evidence for a frontoparietal control system revealed by intrinsic functional connectivity. J Neurophysiol 100:3328-3342.

Vogt BA, Laureys S (2005) Posterior cingulate, precuneal and retrosplenial cortices: cytology and components of the neural network correlates of consciousness. Prog Brain Res 150:205-217.

Vogt BA, Vogt L, Laureys S (2006) Cytology and functionally correlated circuits of human posterior cingulate areas. Neuroimage 29:452-466.

Weissman DH, Roberts KC, Visscher KM, Woldorff MG (2006) The neural bases of momentary lapses in attention. Nat Neurosci 9:971-978.

Wheeler ME, Buckner RL (2004) Functional-anatomic correlates of remembering and knowing. Neuroimage 21:1337-1349.

Zuo XN, Kelly C, Adelstein JS, Klein DF, Castellanos FX, Milham MP (2010) Reliable intrinsic connectivity networks: test-retest evaluation using ICA and dual regression approach. Neuroimage 49:2163-2177. 